depression and of schizophrenia. Mol Psychiatry 2009; July 21. Epub ahead of print.

5 Bullmore E, Fletcher $\mathrm{P}$, Jones $\mathrm{PB}$. Why psychiatry can't afford to be neurophobic. Br J Psychiatry 2009; 194: 293-5.

Nick Craddock, MRC Centre for Neuropsychiatric Genetics and Genomics, Henry Wellcome Building, School of Medicine, Cardiff University, Heath Park, Cardiff CF14 4XN, UK. Email: craddockn@cardiff.ac.uk; Michael J. Owen, MRC Centre for Neuropsychiatric Genetics and Genomics, School of Medicine, Cardiff University, UK

doi: 10.1192/bjp.197.1.75a

Authors' reply: We thank Professors Craddock and Owen for the insightful comments on the possible molecular genetic basis of the relation between epilepsy and psychosis. In most clinical studies of psychosis in patients with epilepsy, individual psychotic vulnerabilities are rarely concerned compared with epilepsyrelated factors. However, several large studies have recently demonstrated genetic vulnerabilities to psychosis even in patients with epilepsy. ${ }^{1,2}$ Our recent work ${ }^{3}$ also demonstrated various factors (i.e. genetic, organic, and epilepsy-related) associated with the development of interictal psychosis in patients with epilepsy.

Psychoses in patients with any central nervous system (CNS) adversity, not only epilepsy but also other brain disorders, can be diagnosed as organic psychosis. The international criteria for mental disorders, either the ICD-10 or the DSM-IV, recognise the traditional dichotomy, i.e. functional and organic psychosis. However, since such CNS adversities are not invariably associated with the development of psychotic states, other additional conditions are required to generate psychotic symptoms. It is known that psychoses after brain injury occur more frequently in people with a family loading of psychoses. ${ }^{4}$ Thus, individual (possibly constitutional) vulnerability to psychosis can be considered as a contributing factor to the development of organic psychosis and its severity.

As for classification systems for mental disorders, many limitations of the Kraepelinian dichotomy between schizophrenia and affective disorders have been discussed. ${ }^{5}$ Likewise, there appear to be limitations to the dichotomous view of organic and non-organic. The concept of organic psychosis has been useful to classify and treat patients, but it appears too simplistic to explain complex pathogenesis in such patients. It may be time to reconceptualise psychoses in patients with or without diagnosable brain disorders.

1 Adachi N, Matsuura M, Hara T, Oana Y, Okubo Y, Kato M, et al. Psychoses and epilepsy: are interictal and postictal psychoses distinct clinical entities? Epilepsia 2002; 43: 1574-82.

2 Qin $\mathrm{P}, \mathrm{Xu} \mathrm{H}$, Laursen TM, Vestergaard M, Moriensen PB. Risk for schizophrenia and schizophrenia-like psychosis among patients with epilepsy: population based cohort study. BMJ 2005; 331: 23-5.

3 Adachi N, Akanuma N, Ito M, Kato M, Hara T, Oana Y, et al. Epileptic, organic and genetic vulnerabilities for timing of the development of interictal psychosis. Br J Psychiatry 2010; 196: 212-6.

4 Corcoran C, Malaspina D. Traumatic brain injury and risk for schizophrenia Int J Mental Health 2001; 30: 17-32.

5 Craddock N, Owen MJ. The Kraepelinian dichotomy - going, going . . . but still not gone. Br J Psychiatry 2010; 196: 92-5.

Naoto Adachi, Adachi Mental Clinic, Kitano 7-5-12, Kiyota, Sapporo 004-0867, Japan Email: adacchan@tky2.3web.ne.jp; Nozomi Akanuma, Department of Psychiatry, National Centre Hospital for Mental, Nervous and Muscular Disorders, Kodaira, and Department of Neuropsychiatry, School of Medicine, Tokyo Medical and Dental University, Tokyo; Masumi Ito, Masaaki Kato, Department of Psychiatry, Nationa Centre Hospital for Mental, Nervous and Muscular Disorders, Kodaira; Tsunekatsu Centre Hospital for Mental, Nervous and Muscular Disorders, Kodaira; Tsunekatsu
Hara, Komagino Hospital, Hachioji; Yasunori Oana, Department of Neuropsychiatry, Hara, Komagino Hospital, Hachioji; Yasunori Oana, Department of Neuropsy
School of Medicine, Tokyo Medical University, Tokyo; Masato Matsuura, Department of Neuropsychiatry, Nihon University School of Medicine, Tokyo; Yoshiro Okubo, Department of Neuropsychiatry, School of Medicine, Tokyo Medical and Dental University, Tokyo; Teiichi Onuma, Department of Psychiatry, National Centre Hospital for Mental, Nervous and Muscular Disorders, Kodaira, Japan

doi: 10.1192/bjp.197.1.76

\section{Heterogeneity of coordinate-based meta-analyses of neuroimaging data: an example from studies in OCD}

Two automated, coordinate-based meta-analyses of voxel-based morphometry (VBM) studies comparing individuals with obsessive-compulsive disorder (OCD) and healthy controls have been recently published, respectively, in the British Journal of Psychiatry $^{1}$ and Neuropsychopharmacology. ${ }^{2}$ Surprisingly, their results are less concordant than one would have expected. We believe this is largely due to methodological differences across the studies.

In coordinate-based meta-analysis, three-dimensional brain maps are built based on the reported coordinates of voxels of peak statistical difference between groups, with higher values being assigned to voxels closer to these coordinates. The full-width at half maximum (FWHM) value of a Gaussian kernel determines the width of spatial distribution, ${ }^{1,3,4}$ thus critically influencing the results. Radua \& Mataix-Cols ${ }^{1}$ used a $25 \mathrm{~mm}$ FWHM kernel, whereas Rotge et $_{\mathrm{al}} \mathrm{l}^{2}$ set this parameter at $12 \mathrm{~mm}$. Such distinction may explain two differences between their results. First, only Radua \& Mataix-Cols reported grey matter increases in the right superior parietal cortex and precuneus, although both studies took exactly the same parietal cortical coordinates $(n=4)$ from the individual VBM investigations. However, these parietal coordinates were not in close proximity to each other, possibly reflecting the spatial uncertainty of OCD-related abnormalities in this area. Since the width of the distribution of voxel values reflects the spatial uncertainty of significant findings, ${ }^{3}$ the greater FWHM kernel used by Radua \& Mataix-Cols possibly afforded greater sensitivity to detect parietal clusters of grey matter difference. Second, although both studies detected striatal foci of increased grey matter, Rotge et al's findings were confined to the putamen, whereas in the study by Radua \& Mataix-Cols these foci spread also to the globus pallidus and caudate nucleus. The greater FWHM value used by Radua \& Mataix-Cols probably explains the lower spatial resolution of the striatal foci in their meta-analysis.

Moreover, Rotge et al used the activation likelihood estimation method, ${ }^{4}$ in which coordinates regarding increased and decreased grey matter are separately computed in independent maps. Conversely, Radua \& Mataix-Cols used the signed differential mapping method, ${ }^{1}$ in which coordinates for findings of either increased or decreased grey matter are reconstructed in the same map, thus influencing each other. Since VBM studies of OCD have identified foci of both increased and decreased grey matter in the orbitofrontal cortex, this may explain why Radua \& Mataix-Cols did not reproduce Rotge et al's finding of grey matter increase in this region of critical relevance to the pathophysiology of OCD. $^{5}$

An additional source of discrepancy relates to the criteria for coordinate selection. Rotge et al included all coordinates reported in the selected studies, regardless of statistical thresholds and correction for multiple comparisons. Conversely, Radua \& Mataix-Cols employed stricter criteria, thus leading to the inclusion of fewer coordinates (as detailed in their article). ${ }^{1}$

In conclusion, these papers are an example of how methodological differences may critically influence the results of coordinate-based meta-analyses. Therefore, when performing such investigations, one should clearly justify the criteria used for coordinate selection and the choice of other methodological parameters. Future studies using such novel techniques should focus on how to foster greater methodological comparability and reproducibility of results.

1 Radua J, Mataix-Cols D. Voxel-wise meta-analysis of grey matter changes in obsessive-compulsive disorder. Br J Psychiatry 2009; 195: 393-402. 